\title{
Euglycemic DKA (euDKA) as a presentation of COVID19
}

\author{
Bhagwan Dass ${ }^{1}$, Andrew Beck ${ }^{2}$, Cody Holmes ${ }^{2}$, and Glenville Morton ${ }^{2}$ \\ ${ }^{1}$ University of Florida \\ ${ }^{2}$ Eglin Air Force Base
}

September 16, 2020

\begin{abstract}
Euglycemic DKA (euDKA) is a serious side effect associated with SGLT2 inhibitors. We present a case euDKA in a patient on an SGLT-2 inhibitor likely precipitated by COVID-19 infection. We suspect that COVID-19 led to euDKA. We pose the question of whether these medications should be discontinued proactively.
\end{abstract}

\section{Keywords:}

COVID-19, diabetic ketoacidosis, euglycemia, diabetes mellitus, diabetes, diabetes type 2, type I diabetes mellitus

\section{Key Clinical Message:}

COVID-19 in the setting of SGLT2 inhibitor use may precipitate euglycemic DKA separate from known acute viral illness and dehydration precipitants. There should be consideration of proactive discontinuation of these medications in these patients.

\section{Introduction :}

Diabetic ketoacidosis (DKA) is a medical emergency characterized by hyperglycemia, metabolic acidosis, and ketosis. EuDKA differs from typical DKA in that it often presents with serious metabolic acidosis but only mild to moderate glucose elevation $(<200 \mathrm{mg} / \mathrm{dl})^{1}$. Known precipitants for euDKA include severe acute illness, dehydration, extreme physical activity, surgery, low carbohydrate intake, fasting, excessive alcohol intake, and SGLT2 inhibitors ${ }^{2}$.

Diabetes is associated with an increased risk of severe COVID-19 with both higher morbidity and mortality rates in patients with diabetes mellitus ${ }^{3,4}$. SARS-CoV-2 utilizes the ACE-2 receptor for viral entry, which is expressed in several organs, and may have diabetogenic effects beyond the well-recognized stress response associated with severe illness. The virus may cause alterations of glucose metabolism at the tissue level directly and indirectly that could complicate existing diabetes ${ }^{5}$. COVID-19 may alter the pathophysiology of preexisting diabetes or worsen it with associated complications such as ketoacidosis.

\section{Case Presentation :}

A 59 year-old female with history of documented type 2 diabetes on empagliflozin, sitagliptin, and metformin presented with 9 days of progressively worsening shortness of breath, low grade fevers, and fatigue. She was seen two days prior in the emergency department and had an elevated glucose of 198, normal CO2 of 22 (normal 20-31) on basic metabolic panel and was found to have bilateral infiltrates on chest x-ray. She was diagnosed with community acquired pneumonia and discharged on doxycycline. On representation to the ED less than 48 hours later, she presented with tachypnea, tachycardia, and was found to have a profound metabolic acidosis with significant respiratory compensation with an associated non-gap acidosis as seen on 
her initial $\mathrm{ABG}(\mathrm{pH}$ of $6.94, \mathrm{PaCO} 2$ of $13, \mathrm{PaO} 2$ of 99 and a $\mathrm{HCO} 3$ of 3). On serum analysis her lactate was 0.9 , her glucose 154, confirmed bicarb of $<10$, serum osmolality of 346 , an elevated anion gap of 30 , beta-hydroxybutyrate of 95 . Her urinalysis showed $3+$ glucose and $2+$ ketones. She had a negative UDS and salicylate levels were normal. She was found to have a positive COVID-19 test and was admitted for Euglycemic DKA (euDKA). She was started on an insulin drip and IV fluid and had resolution over the next 2 days. After resolution of DKA the patient was continued on her Sitagliptin and Metformin, but Empagliflozin was discontinued given the association of SGLT2 inhibitors with euDKA. She was also started on 20 units of insulin glargine nightly which was continued upon discharge.

\section{Discussion :}

We performed a literature search of PubMed using a combination of the words "euglycemic diabetic ketoacidosis," "COVID-19" with "SGLT2 inhibitors." To our knowledge this is the first case report in the literature that documents DKA with normal glucose levels in the setting of COVID-19 and SGLT2 inhibitor use in type 2 Diabetes (there is a case report in type 1 ).

Diabetic ketoacidosis (DKA) is a medical emergency characterized by hyperglycemia (blood sugar $>250$ $\mathrm{mg} / \mathrm{dl}$ ), metabolic acidosis (arterial $\mathrm{pH}<7.3$ and serum bicarbonate $<18 \mathrm{mEq} / \mathrm{L}$ ), and ketosis - Euglycemic DKA includes blood glucose levels $<200 \mathrm{mg} / \mathrm{dl}$, arterial $\mathrm{pH}<7.3$, anion gap $>12 \mathrm{mEq} / \mathrm{L}, \mathrm{HCO} 3-<15$ $\mathrm{mEq} / \mathrm{L}$ and the presence of ketones in blood and urine ${ }^{1}$. EuDKA was first described as a discrete entity by Munro et al. in $1973^{6}$. EuDKA differs from typical DKA in that it often presents with serious metabolic acidosis but only mild to moderate glucose and anion gap elevation. Diagnosis should be confirmed with the direct measurement of the beta-hydroxybutyrate level in blood and arterial blood $\mathrm{pH}^{7}$. Known precipitants for euDKA include severe acute illness, dehydration, extreme physical activity, surgery, low carbohydrate intake, fasting, excessive alcohol intake, and SGLT2 inhibitors ${ }^{2}$.

The increased risk of euDKA associated with SGLT2 inhibitors is well known. In 2015, the FDA released safety warnings about the risk of euDKA associated with SGLT2 inhibitors ${ }^{8}$. The development of euDKA with SGLT2 inhibitors is thought to involve decreased insulin production and increase in secretion of glucagon. Increase in glucagon levels is multifactorial including both direct and indirect mechanisms. It is increased directly via effects on SGLT2 expressed in the glucagon-secreting alpha pancreatic cells. SGLT2 inhibitors also increase glucose excretion leading to relative lower levels of insulin and low ratio of insulin to glucagon. The relative lack of insulin stimulates the production of free fatty acids and ketone bodies and a shift from glucose to fat metabolism causing ketoacidosis ${ }^{9}$.

Ketone production may be further stimulated in the setting of SGLT2 inhibitors by the lowering of glucose reabsorption in the proximal tubules increasing glycosuria and possibly simulating starvation conditions ${ }^{10}$. Ketones can be excreted in the urine as sodium salts and are essentially the equivalent to the loss of bicarbonate causing metabolic acidosis ${ }^{11}$.

In addition to SGLT2 inhibitors, the COVID-19 virus itself may be directly linked to the development of euDKA. Studies have shown that COVID-19 utilizes the ACE-2 receptor, which is expressed on human pancreatic beta cells, to gain entry to and infect human cells. Once inside the cell, an immune response is triggered which leads to the production of cytokines and chemokines resulting directly in cell death ${ }^{12}$. A similar effect was seen in the similar SARS-CoV-1 coronavirus in which the virus also utilized the ACE2 receptor in the islet cells of the pancreas to gain entry leading to direct cellular destruction precipitating acute diabetes in a subset of patients ${ }^{5}$. Acute infection with COVID-19 could lead to further decrease in insulin production, and predisposition to euDKA. Low $\mathrm{pH}$ in DKA also favors the entry and replication of the SARS-CoV-2, and development of DKA makes the disease course of COVID-19 worse $^{13}$.

In this case the development of euDKA was likely multifactorial in the setting of COVID-19 infection and SGLT2 inhibitor use and it is likely that COVID-19 was an inciting factor for euDKA. Our patient who was on metformin and empagliflozin prior to COVID-19 infection ended up being discharged on insulin most likely due to combination of COVID-19 illness and impaired beta cell function in setting of SARS-CoV-2 Infection. 
Physicians need to acquaint themselves with euDKA to promptly recognize and treat this medical emergency. This is particularly imperative in this pandemic of COVID-19 and wide-spread use of SGLT2 inhibitors given their proven cardiovascular and renal benefits. We believe the risk of SGLT2-inhibitor-associated euDKA can be reduced or prevented by the discontinuation of these medications during acute illness with COVID-19

As the majority of COVID-19 patients with type 2 diabetes are managed as an outpatient with home quarantine, one possible option is to stop SGLT2 inhibitors or reduce their dose.

As research is evolving there could be a potential role for dipeptidyl peptidase 4 (DPP4)-inhibitors. DPP4inhibitors might interfere with and modify viral binding and hypothetically reduce virulence. DPP4inhibitors modulate inflammation and exert anti-fibrotic activity. These properties may be of potential use for halting progression to the hyper inflammatory state associated with severe COVID-1914. This interaction deserves further investigation and possibly DPP4-inhibitor trial during active COVID-19 infection.

\section{References:}

1. Rawla, Prashanth et al. "Euglycemic diabetic ketoacidosis: a diagnostic and therapeutic dilemma." Endocrinology, diabetes $\& 3$ metabolism case reports vol. 2017 17-0081. 4 Sep. 2017, doi:10.1530/EDM17-0081

2. Goldenberg, Ronald M et al. "SGLT2 Inhibitor-associated Diabetic Ketoacidosis: Clinical Review and Recommendations for Prevention and Diagnosis." Clinical therapeutics vol. 38,12 (2016): 26542664.e1. doi:10.1016/j.clinthera.2016.11.002

3. Roncon, Loris et al. "Diabetic patients with COVID-19 infection are at higher risk of ICU admission and poor short-term outcome." Journal of clinical virology : the official publication of the Pan American Society for Clinical Virology vol. 127 (2020): 104354. doi:10.1016/j.jcv.2020.104354

4. Yan, Yongli et al. "Clinical characteristics and outcomes of patients with severe covid-19 with diabetes." BMJ open diabetes research \& care vol. 8,1 (2020): e001343. doi:10.1136/bmjdrc-2020-001343

5. Yang, Jin-Kui et al. "Binding of SARS coronavirus to its receptor damages islets and causes acute diabetes." Acta diabetologica vol. 47,3 (2010): 193-9. doi:10.1007/s00592-009-0109-4

6. Munro, J F et al. "Euglycaemic diabetic ketoacidosis." British medical journal vol. 2,5866 (1973): 578-80. doi:10.1136/bmj.2.5866.578

7. Vanelli, $\mathrm{M}$ et al. "The direct measurement of 3-beta-hydroxy butyrate enhances the management of diabetic ketoacidosis in children and reduces time and costs of treatment." Diabetes, nutrition $\mathcal{E}^{3}$ metabolism vol. 16,5-6 (2003): 312-6.

8. "FDA Warns That SGLT2 Inhibitors for Diabetes May Result in a Serious Condition of Too Much Acid in the Blood." FDA.gov , 2015, www.fda.gov/media/92185/download.

9. Taylor, Simeon I et al. "SGLT2 Inhibitors May Predispose to Ketoacidosis." The Journal of clinical endocrinology and metabolism vol. 100,8 (2015): 2849-52. doi:10.1210/jc.2015-1884

10. Qiu, Hongyu et al. "Ketosis and diabetic ketoacidosis in response to SGLT2 inhibitors: Basic mechanisms and therapeutic perspectives." Diabetes/metabolism research and reviews vol. 33,5 (2017): 10.1002/dmrr.2886. doi:10.1002/dmrr.2886

11. Sampani, Erasmia et al. "Severe euglycemic diabetic ketoacidosis of multifactorial etiology in a type 2 diabetic patient treated with empagliflozin: case report and literature review." BMC nephrology vol. 21,1 276. 15 Jul. 2020, doi:10.1186/s12882-020-01930-6

12. Yang et al., 2020, Cell Stem Cell 27, 125-136 July 2, 2020 a 2020 Elsevier Inc. https://doi.org/10.1016/j.stem.2020.06.015

13. Muniyappa, Ranganath, and Sriram Gubbi. "COVID-19 pandemic, coronaviruses, and diabetes mellitus." American journal of physiology. Endocrinology and metabolism vol. 318,5 (2020): E736-E741. doi:10.1152/ajpendo.00124.2020

14. Iacobellis, Gianluca. "COVID-19 and diabetes: Can DPP4 inhibition play a role?." Diabetes research and clinical practice vol. 162 (2020): 108125. doi:10.1016/j.diabres.2020.108125

Consent : Informed consent was obtained from patient for the study. 


\section{Author contributions :}

$\mathrm{BD}, \mathrm{AB}, \mathrm{CH}$ wrote manuscript, revised and approved manuscript, $\mathrm{MG}, \mathrm{AB}, \mathrm{CH}$ involved in patient management, revised manuscript, approved manuscript

\section{Conflict of interest -}

The authors declare that they have no conflicts of interest.

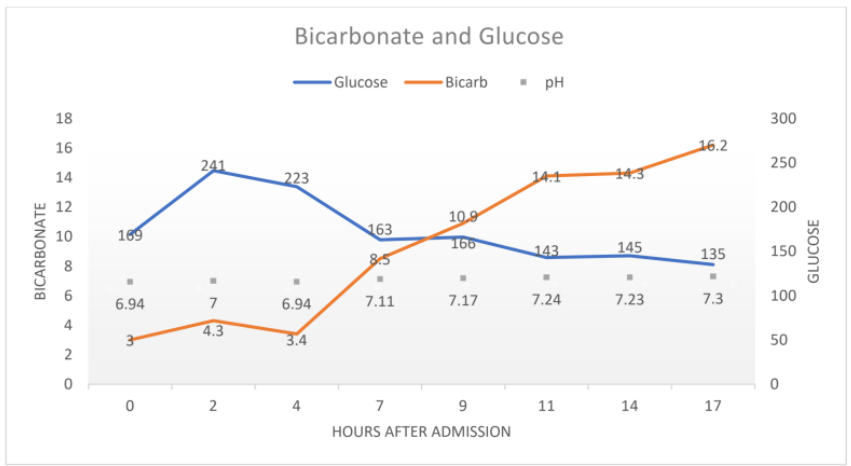

Figure 1: Laboratory values over the initial course of hospitalization 


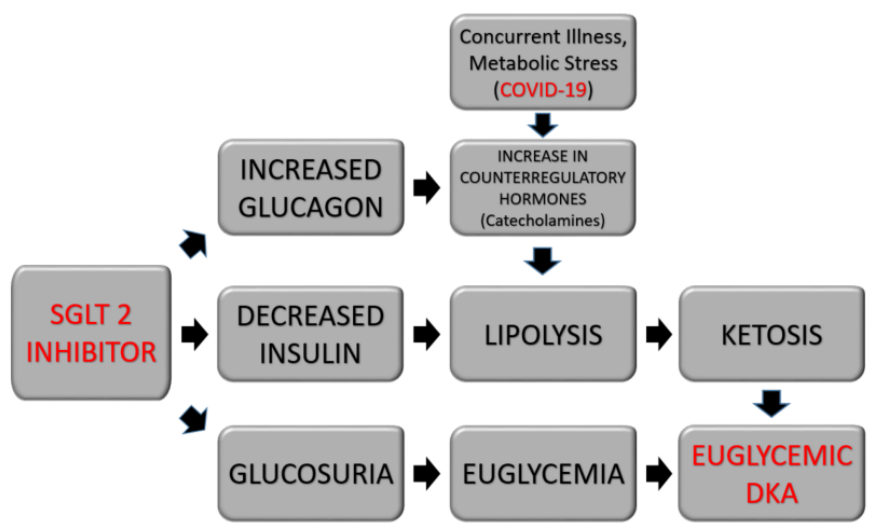

Figure 2: Role of SGLT 2 inhibitors and COVID-19 in the development of euglycemic DKA 